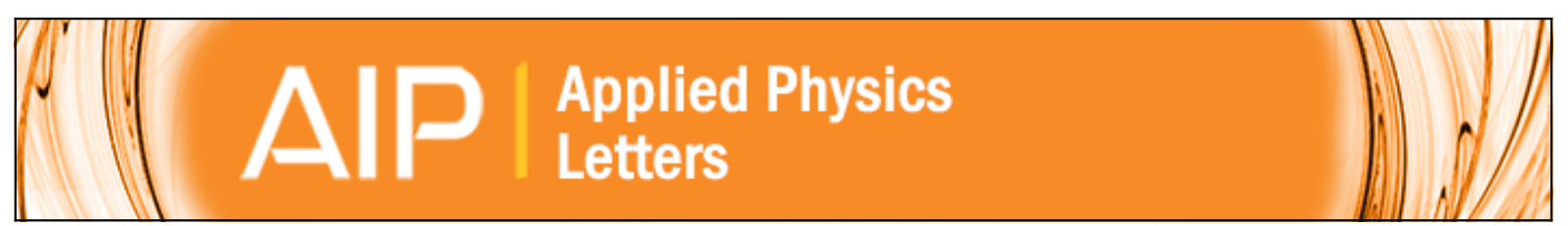

Inducing bistability with local electret technology in a microcantilever based non-linear vibration energy harvester

M. López-Suárez, J. Agustí, F. Torres, R. Rurali, and G. Abadal

Citation: Applied Physics Letters 102, 153901 (2013); doi: 10.1063/1.4800926

View online: http://dx.doi.org/10.1063/1.4800926

View Table of Contents: http://scitation.aip.org/content/aip/journal/apl/102/15?ver=pdfcov

Published by the AIP Publishing

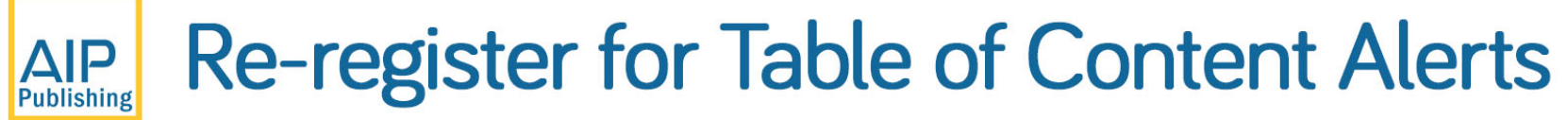

Create a profile. 


\title{
Inducing bistability with local electret technology in a microcantilever based non-linear vibration energy harvester
}

\author{
M. López-Suárez, ${ }^{1, a)}$ J. Agustí, ${ }^{1}$ F. Torres, ${ }^{1}$ R. Rurali, ${ }^{2}$ and G. Abadal ${ }^{1}$ \\ ${ }^{1}$ Departament d'Enginyeria Electrònica, Universitat Autònoma de Barcelona, Bellaterra 08193, Spain \\ ${ }^{2}$ Institut de Ciència de Materials de Barcelona (ICMAB-CSIC), Campus de Bellaterra, Bellaterra 08193 , \\ Spain
}

(Received 22 January 2013; accepted 21 March 2013; published online 16 April 2013)

\begin{abstract}
A micro-electro-mechanical system based vibration energy harvester is studied exploring the benefits of bistable non linear dynamics in terms of energy conversion. An electrostatic based approach to achieve bistability, which consists in the repulsive interaction between two electrets locally charged in both tip free ends of an atomic force microscope cantilever and a counter electrode, is experimentally demonstrated. A simple model allows the prediction of the measured dynamics of the system, which shows an optimal distance between the cantilever and the counter electrode in terms of the root mean square vibration response to a colored Gaussian excitation noise. (C) 2013 American Institute of Physics. [http://dx.doi.org/10.1063/1.4800926]
\end{abstract}

Among all the explored alternatives to improve the performance characteristics in vibration energy harvesting (VEH) schemes, those based on the non-linear mechanical properties of the transducer element have demonstrated to be one of the most promising options. ${ }^{1-3}$ In particular, two main issues make these systems to perform better than traditional linear ones. First, they are able to harvest energy from wide band non-harmonic vibration sources; consequently, they do not require complex tuning strategies in the transducer to match the characteristic frequency of the energy source. ${ }^{4}$ Second, they can extract energy from low frequency sources in a non-resonant way. Therefore large inertial masses and large volume realizations are not strictly needed. ${ }^{5}$

On the one hand, bistable implementations have become one of the most reported non-linear vibration energy harvesting (NLVEH) choices. Several ways to induce bistability in NLVEH have been demonstrated so far: magnetic repulsion, ${ }^{2}$ inverted cantilever, ${ }^{6}$ induced or residual stress. ${ }^{7}$

On the other hand, it has been demonstrated that microelectro-mechanical systems (MEMS) technology can provide solutions for NLVEH with improvements in the energy level sensitivity, ${ }^{8}$ according to their extremely high mechanical compliance.

However, in most of the previous cases, a physical realization of the transducer at the nano or even at the microscale becomes technologically difficult, due to the low control of the bistability parameter during the fabrication process (stress) or due to material non-compatibility (magnetic materials).

In this paper, we demonstrate an alternative based on local electret technology to induce bistability in the mechanical behavior of a triangular tipless AFM-like microcantilever. A simple method based on the capacitance constant voltage stress is used to locally charge the free end of a silicon nitride cantilever. A counter electrode (CE), having the same geometry and material as the cantilever, is also locally charged by the same procedure and brought in close proximity to the cantilever. Repulsive electrostatic interaction

\footnotetext{
${ }^{\text {a) }}$ Author to whom correspondence should be addressed. Electronic mail: miquel.lopez@uab.cat
}

between both electrets combined with the restoring force of the cantilever gives rise to a bistable potential for a certain cantilever-electrode distance, which is used as the parameter to control the bistability. A detailed study of the cantilever dynamics when it is excited with a colored vibration noise is presented for different bistability and noise intensity conditions.

We consider a commercial $600 \mathrm{~nm}$ thick silicon nitride $\left(\mathrm{Si}_{3} \mathrm{~N}_{4}\right) \mathrm{V}$-shaped cantilever, ${ }^{9}$ as the one depicted in the top side of Figure 1(a) with a $65 \mathrm{~nm}$ thick $\mathrm{Cr} / \mathrm{Au}$ layer coating the backside of the structure.

The AFM-like cantilever has a nominal resonance frequency and a spring constant of $17 \mathrm{kHz}$ and $0.08 \mathrm{~N} / \mathrm{m}$, respectively. The same kind of triangular structure, but having a length short enough to prevent any significant displacement, is used to define a counter electrode (see bottom side of Figure 3(a)).

The tip of the cantilever or the counter electrode are independently charged by applying a voltage ( $15 \mathrm{~V}$ typically during $30 \mathrm{~min}$ ) between the $\mathrm{Cr} / \mathrm{Au}$ coated top side and the back sides through a contacting gold coated sample, as shown in Figure 2(a). During this process, some charges overcome the barrier at the metal-insulator junction and end up trapped at the surface or deep in the body of the cantilever/electrode. ${ }^{10}$ To detect the dynamics of the system we use a conventional AFM optical readout setup, as described in Figure 2(b). The alignment of the cantilever and the counter electrode is achieved by moving both elements with one each 3D micropositioners. Top and lateral views are obtained by means of two CCD cameras which provide an optical feedback during the whole process. A precise control of the cantilever-CE gap distance, $\mathrm{d}$, is achieved with a long range piezo-stack which produces a longitudinal displacement of the $\mathrm{CE}$ at a $0.1 \mu \mathrm{m} / \mathrm{V}$ ratio. The deflection of a reflected $\mathrm{HeNe}$ laser beam $(632.8 \mathrm{~nm}, 15 \mathrm{~mW})$ on the cantilever surface is detected by means of a position sensitive detector (PSD, New Focus 2930). The transduced electrical signal is captured and stored through an oscilloscope. The mechanical excitation of the cantilever is done through a piezoelectric shaker connected to a voltage noise generator. We have 


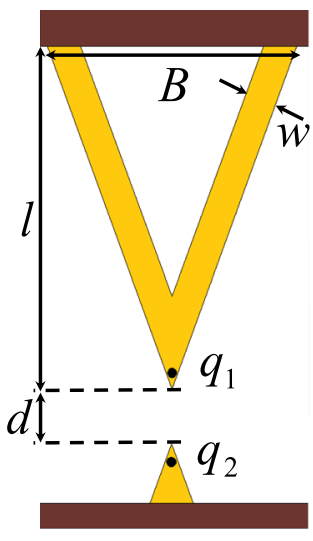

(a)

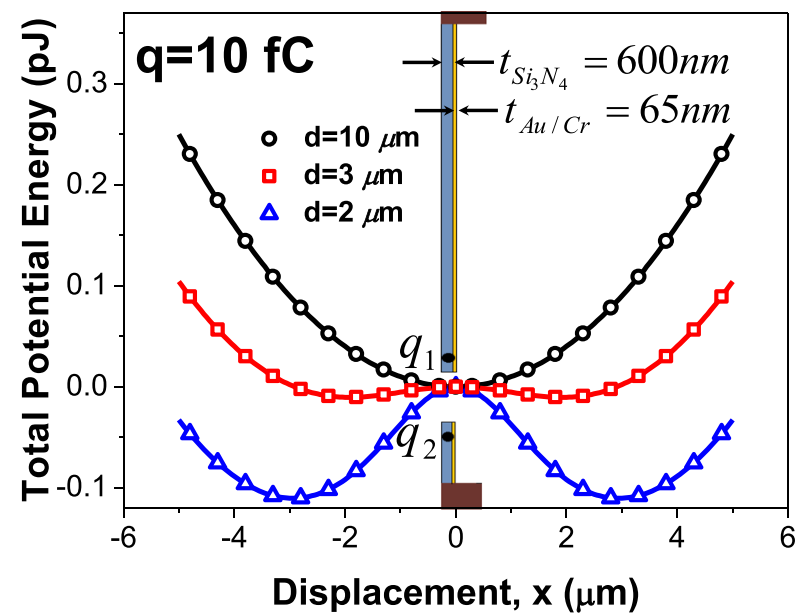

(b)
FIG. 1. (a) Scheme of the experimental system. A V-shaped cantilever (top) $(1=200 \mu \mathrm{m}$; $\mathrm{w}=28 \mu \mathrm{m} ; \mathrm{B}=184 \mu \mathrm{m})$ and a counter electrode (bottom) are permanent and locally charged with $\mathrm{q}_{1}$ and $\mathrm{q}_{2}$, respectively, at their free ends and separated by a distance d. (b) Total potential energy for different $\mathrm{d}$ values showing the transition from monostability to bistability as this distance is reduced. For the sake of simplicity an effective electric charge is defined as $\mathrm{q}=\left(\mathrm{q}_{1} \cdot \mathrm{q}_{2}\right)^{1 / 2}$. Inset in (b) is a view of the system indicating the thickness of the composite structure. verified that the noise used to excite the device is a pink noise, with the intensity inversely proportional to the frequency, as expected in $1 / \mathrm{f}$ noise.

We assume a simple elastic restoring force, $\mathrm{F}_{\mathrm{el}}=-\mathrm{k} \cdot \mathrm{x}$, for the cantilever, where $\mathrm{k}$ and $\mathrm{x}$ are the stiffness and the out-of-plane displacement, respectively. A nominal value of $\mathrm{k}=0.08 \mathrm{~N} / \mathrm{m}$ is considered.

We assume that the electric charges, $\mathrm{q}_{1}$ and $\mathrm{q}_{2}$, have the same sign and that they are trapped at the tip end of the cantilever and CE. Facing them as shown in Figure 1, we can compute the total potential energy of the system as

$$
\mathrm{V}(\mathrm{x})=1 / 2 \cdot \mathrm{k} \cdot \mathrm{x}^{2}+\mathrm{K} \cdot \mathrm{q}^{2} /\left(\mathrm{d}^{2}+\mathrm{x}^{2}\right)^{1 / 2},
$$

where $\mathrm{K}$ is the Coulomb constant and $\mathrm{q}=\left(\mathrm{q}_{1} \cdot \mathrm{q}_{2}\right)^{1 / 2}$. This defines a bistable potential for certain values of the parameters k, q, and d as it can be seen in Figure 1(b). Deriving and solving the equation $\mathrm{dV} / \mathrm{dx}=0$, the position of the stable points can be found as well as the potential barrier height in terms of these three parameters

$$
\begin{gathered}
\mathrm{x}_{\min }=\left(\left(\mathrm{Kq}^{2} / \mathrm{k}\right)^{2 / 3}-\mathrm{d}^{2}\right)^{1 / 2}, \\
\Delta \mathrm{V}=\mathrm{V}(0)-\mathrm{V}\left(\mathrm{x}_{\min }\right) .
\end{gathered}
$$

It is clear that to achieve the bistability condition, expression (2) must give a real number and, then, one can express this condition as $\mathrm{d}<\left(\mathrm{Kq}^{2} / \mathrm{k}\right)^{1 / 3}$. As it is expected, this range of values assure a real value for the barrier height as well. For the experimental purposes, the nonlinearizing parameter will be the distance between electric charges as both $\mathrm{q}$ and $\mathrm{k}$ will be determined before displaying the experiment while $\mathrm{d}$ will be a tunable quantity.

Figure 3(a) (bottom) shows the trajectory followed by the system when driven by a Colored Gaussian noise for three different values of the distance between charges, $d$,

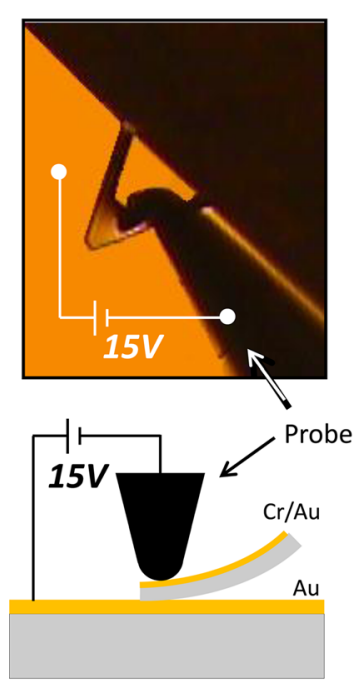

(a)

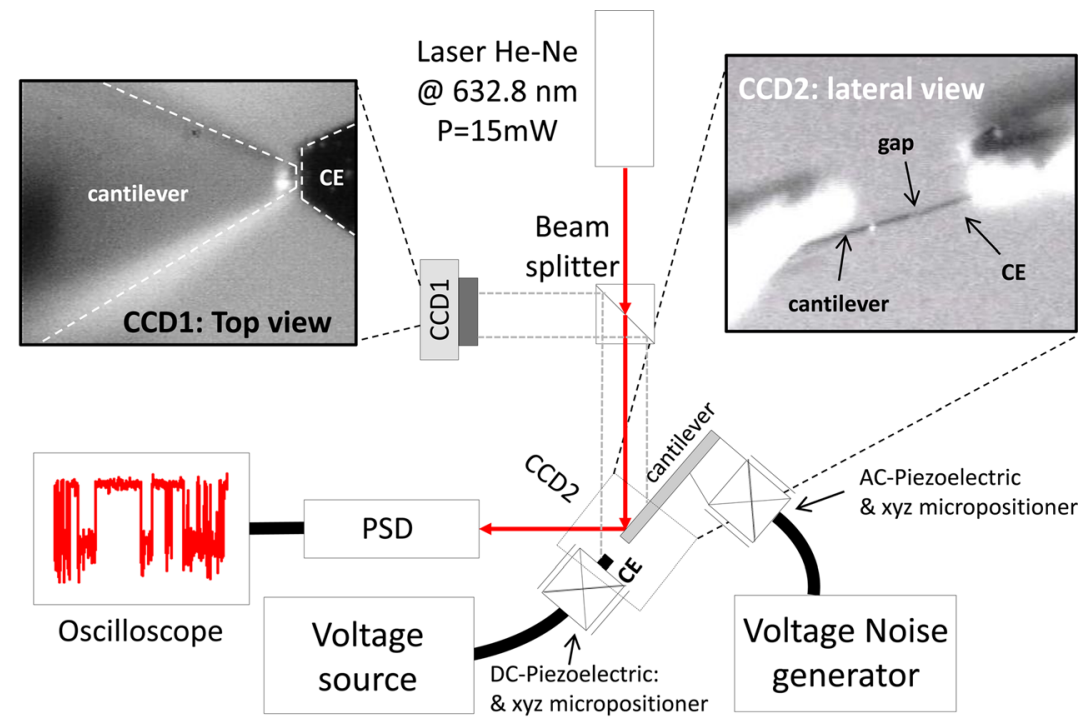

(b)

FIG. 2. (a) Optical image (top) and scheme (bottom) of the electrets charging setup/procedure. A probe is used to apply a voltage to the $\mathrm{Cr} / \mathrm{Au}-\mathrm{Si}{ }_{3} \mathrm{~N}_{4}-\mathrm{Au}$ capacitor formed by a gold coated sample and the cantilever (or counter electrode). (b) Measurement setup: the deflection of a laser beam incident to the cantilever surface is detected by a PSD in terms of voltage. Two optical images of the system show the captures of the two CCD's which allow the cantilever-CE alignment through two 3D micro-positioners. The finer control of $\mathrm{d}$ is carried out through a DC piezoelectric stack with a characteristic ratio of $0.1 \mu \mathrm{m} / \mathrm{V}$. 


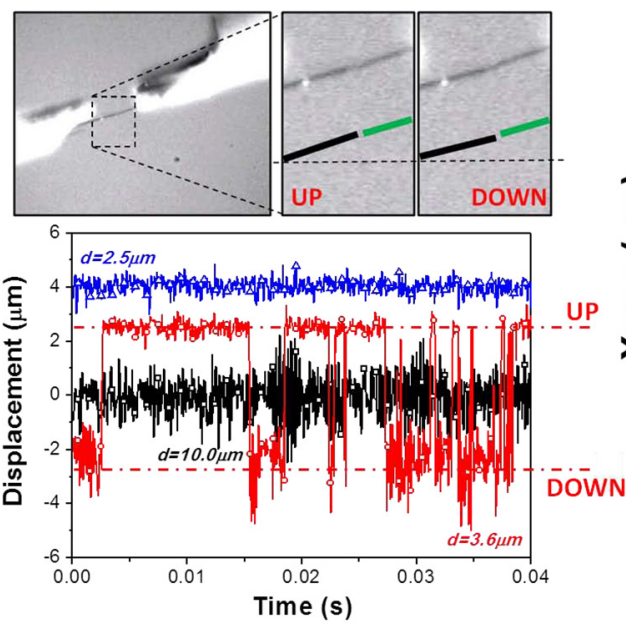

(a)

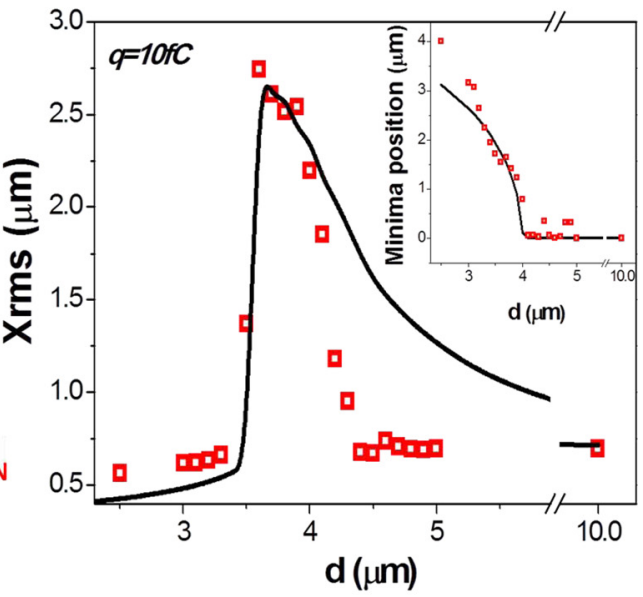

(b)

FIG. 3. (a) Upper side: lateral optical image and drawing showing the "up" and "down" states of the cantilever (left, black) movement, with respect to the fixed $\mathrm{CE}$ (right, green). Lower side: different regimes of the dynamical response of the system for three different values of the distance between the charges, d: monostability around $\mathrm{x}=0$ (black squares), bistability (red circles), and monostability around $\mathrm{x}=\mathrm{x}_{+}$(blue triangles). (b) Experimental (symbols) and simulated (lines) results for the $x_{r m s}$ and potential minima position $x_{\min }=x_{+}$as a function of $d$. The dynamics of the system is obtained solving $m_{\text {eff }} \cdot d^{2} x / d^{2}$ $=-\mathrm{b} \cdot \mathrm{dx} / \mathrm{dt}-\mathrm{dV} / \mathrm{dx}+\mathrm{F}_{\mathrm{ext}} \mathrm{x}(\mathrm{t})$, where $\mathrm{x}$ stands for the displacement, $\mathrm{b}$ represents the energy losses of the system, and $\mathrm{F}_{\text {ext }} \mathrm{x}(\mathrm{t})$ represents the external force as a stochastic process with intensity $\mathrm{F}_{\mathrm{ext}}$. The noise intensity is given by $\mathrm{F}_{\mathrm{rms}}=4 \mathrm{nN}$ (enhanced online) [URL: http://dx.doi.org/10.1063/1.4800926.1].

representative of the three characteristic dynamical regimes. (i) For large d, the system responds as a linear resonant system oscillating around $\mathrm{x}=0$ (black line). (ii) As d decreases and charges get closer, the bistability appears and the cantilever is able to jump from one potential minimum to the other in a random sequence (red line). (iii) Finally, when charges are too close, the potential barrier is too high and the dynamics gets stuck in one of the two wells: the cantilever oscillates around one of its equilibrium positions, but lowerfrequency swings to the other minimum are hindered by the barrier height (blue line). An optical image of the system is shown in the upper panel of Figure 3(a), where magnified images slightly allow differentiating the up and down states of the bistable regime. This can be seen more clearly in the video provided (see link in Fig. 3 caption). Besides, note that in top Figure 3(a) images, the optical resolution and the lack of perfect perpendicularity of the view avoid to solve both the $600 \mathrm{~nm}$ thickness of the cantilever (or of the CE) and the gap distance. In Figure 3(b) we plot the displacement root mean square, $\mathrm{x}_{\mathrm{rms}}$, obtained experimentally (red symbols) for different values of the distance $d$, while keeping fixed the noise intensity. These results are in good agreement with the predictions of the model (continuous lines). Clearly, there is an optimal cantilever-CE gap, $\mathrm{d}_{\mathrm{opt}}=3.6 \mu \mathrm{m}$, that maximizes the system response in terms of $x_{\mathrm{rms}}$, which is closely related to the capability of transducing kinetic energy into electric energy. ${ }^{7}$ The position of the potential energy minima as a function of d (inset of Figure 3(b)), obtained by measuring the average position, also shows a good agreement between experimental data and model (Eq. (2)).

Thus far, we have considered a given noise intensity and optimized the separation between charges, achieving the bistability and maximizing the root mean square of the cantilever displacement. Now we consider the case of a given value of $d$, and we show that, as expected, there is always a value for the noise intensity from which the system again becomes strongly non-linear, jumping from one well to the other, while below this threshold the cantilever gets stuck in one of the two wells. This behavior is shown in Figure 4, pointing out that the benefits of bistability can be extended to all $\mathrm{F}_{\mathrm{rms}}$ below the rupture limit when overcoming a threshold value. Furthermore, even beneath this threshold value, when the external noise is not able to provoke transitions over the potential barrier, the response seems to be of the same order of that for the resonating case.

In summary, we have modeled, fabricated, and tested a bistable NLVEH where a strategy based on local electrets technology is used to engineer the non-linearity of the system. The harvester, consisting in a $0.08 \mathrm{~N} / \mathrm{m} \mathrm{Si}_{3} \mathrm{~N}_{4}$ microcantilever faced to a counter electrode, is driven into bistable regime by controlling the distance between the tip end of both elements, where two electric charges of the same sign have been implanted, thus tuning their electrostatic repulsion. A good agreement between the predictions of a simple

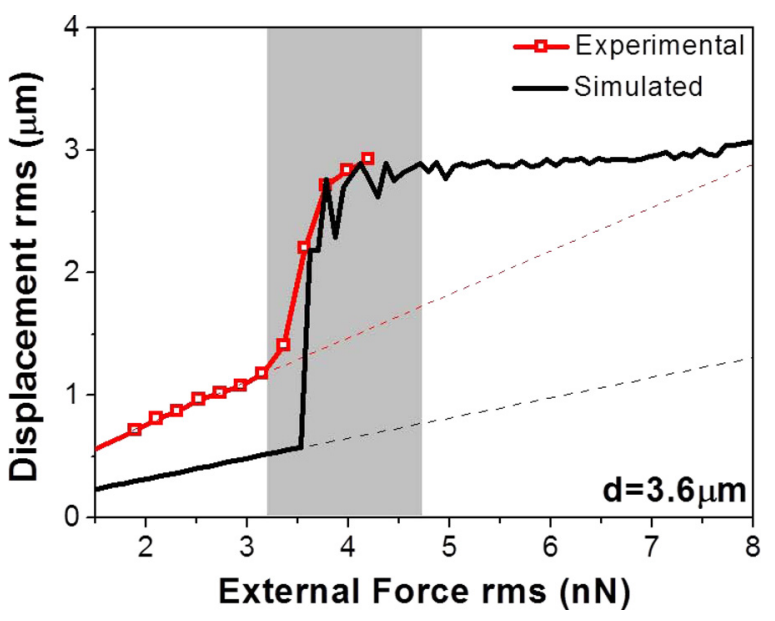

FIG. 4. Experimental (red line-symbols) and simulated (black line) results for the $\mathrm{x}_{\mathrm{rms}}$ for a given distance $\mathrm{d}$ as the noise intensity is swept showing an abrupt increment when a threshold value is overcome. The dashed line represents the trend of this quantity if no bistability is considered, i.e., $q=0$. 
model and the experimental data is achieved reproducing all the main features of the expected dynamics. The proof-ofconcept demonstrated in this letter should now be followed by a careful optimization of the device parameters, including the material of choice. While here we have used commercial $\mathrm{Si}_{3} \mathrm{~N}_{4}$ cantilevers, other materials such as Teflon should be preferred for the much longer decay times of the trapped charge. ${ }^{11,12}$

The authors would like to thank the Spanish "Ministerio de Ciencia y Innovación" for financially supporting this research under the projects OPACMEMS Nos. ENE20091430-CO2-02, FIS2009-12721-C04-03, and FIS2012-37549C05-05.

${ }^{1}$ L. Gammaitoni, H. Vocca, I. Neri, F. Travasso, and F. Orfei, Vibration Energy Harvesting: Linear and Nonlinear Oscillator Approaches,
Sustainable Energy Harvesting Technologies - Past, Present and Future (Dr. Yen Kheng Tan, Shanghai, 2011), pp. 169-188.

${ }^{2}$ F. Cottone, H. Vocca, and L. Gammaitoni, Phys. Rev. Lett. 102, 080601 (2009).

${ }^{3}$ L. Gammaitoni, I. Neri, and H. Vocca, Appl. Phys. Lett. 94, 164102 (2009).

${ }^{4}$ M. Lallart, S. R. Anton, and D. J. Inman, J. Intell. Mater. Syst. Struct. 21, 897-906 (2010).

${ }^{5}$ G. Murillo, G. Abadal, F. Torres, J. L. López, J. Giner, A. Uranga, and N. Barniol, Microelectron. Eng. 86, 1183 (2009).

${ }^{6}$ M. I. Friswell, S. F. Ali, O. Bilgen, S. Adhikari, A. W. Lees, and G. Litak, J. Intell. Mater. Syst. Struct. 23(13), 1505 (2012).

${ }^{7}$ F. Cottone, L. Gammaitoni, H. Vocca, M. Ferrari, and V. Ferrari, Smart Mater. Struct. 21, 035021 (2012).

${ }^{8}$ M. López-Suárez, R. Rurali, and G. Abadal, Phys. Rev. B 84, 161401(R) (2011).

${ }^{9}$ NanoWorld ${ }^{\mathrm{TM}}$ Pirex Nitride AFM probe: PNP-TR-TL.

${ }^{10}$ J. Wibbeler, G. Pfeifer, and M. Hietschold, Sens. Actuators, A 71, 74 (1998).

${ }^{11}$ P. W. Chudleigh, R. E. Collins, and G. D. Hancock, Appl. Phys. Lett. 23, 211 (1973).

${ }^{12}$ J. A. Małecki, Phys. Rev. B 59, 9954 (1999). 